\title{
Variation of the electronic dipole polarizability on the reaction path
}

\author{
Mateusz Jędrzejewski • Piotr Ordon • \\ Ludwik Komorowski
}

Received: 6 November 2012 / Accepted: 20 February 2013 / Published online: 23 March 2013

(C) The Author(s) 2013. This article is published with open access at Springerlink.com

\begin{abstract}
The reaction force and the electronic flux, first proposed by Toro-Labbé et al. (J Phys Chem A 103:4398, 1999) have been expressed by the existing conceptual DFT apparatus. The critical points (extremes) of the chemical potential, global hardness and softness have been identified by means of the existing and computable energy derivatives: the Hellman-Feynman force, nuclear reactivity and nuclear stiffness. Specific role of atoms at the reaction center has been unveiled by indicating an alternative method of calculation of the reaction force and the reaction electronic flux. The electron dipole polarizability on the IRC has been analyzed for the model reaction $\mathrm{HF}+\mathrm{CO} \rightarrow \mathrm{HCOF}$. The electron polarizability determined on the IRC $\alpha_{e}(\xi)$ was found to be reasonably parallel to the global softness curve $S(\xi)$. The softest state on the IRC (not TS) coincides with zero electronic flux.
\end{abstract}

Keywords Electron dipole polarizability $\cdot$ Mode softening index $\cdot$ Nuclear reactivity $\cdot$ Nuclear stiffness $\cdot$ Reaction electronic flux $\cdot$ Reaction force $\cdot$ Softest state

\section{Introduction}

Toro-Labbé and coworkers have presented a fresh and interesting concept by demonstrating how the specific derivatives

M. Jędrzejewski $\cdot$ L. Komorowski $(\square)$

Institute of Physical and Theoretical Chemistry,

Wrocław University of Technology, Wyb. Wyspiańskiego 27,

50-370 Wrocław, Poland

e-mail: Ludwik.Komorowski@pwr.wroc.pl

P. Ordon

Physics and Biophysics Laboratory, Wrocław University

of Environmental and Life Sciences, ul. Norwida 25,

50-373 Wrocław, Poland computed on the IRC change with evolution of the reaction [1-5]:

Reaction force : $F_{\xi}=-\frac{d E}{d \xi}$

Reaction electronic flux : $J_{\xi} \propto \frac{d \mu}{d \xi}$,

$E$ stands for the electronic energy of the system, $\mu=(\partial E /$ $\partial N)_{v}$ is the chemical potential of electrons and $\xi$ is the progress of the reaction on the IRC. Other authors studied the reaction profiles for the global hardness: $\eta=\left(\partial^{2} E / \partial N^{2}\right)_{v}$. Torrent-Sucarrat et al. [6] first found the non-symmetric stationary points for the hardness profiles in simple reactions; the same group also studied the hardness modification through the molecular distortions along vibrational modes of polycyclic hydrocarbons [7]. The hardness stationary points discovered were less sensitive to the calculation technique than those for the energy profile. Ordon and Tachibana presented an analysis for the profile of the molecular stiffness on the reaction path [8]: $G_{\xi}=d \eta / d \xi$. These authors also presented an effort to determine the electronic flux between the regions in the reacting entity [9].

The conceptual density functional theory has developed a variety of concepts [10] to characterize the electronic systems by taking the energy as a function of a number of electrons $N$ and as a functional of the external potential [11] $v(\mathbf{r})$. The role of the specific external potential from the nuclei of a chemical entity has been analyzed by Ordon and Komorowski [12]. The authors analyzed the variety of energy derivatives over the nuclear displacement $\left(R_{i}\right)[13$, 14]. Since the IRC analysis [15] operates with the variations of the atomic positions, the appropriate derivatives of the electronic energy over $R_{i}$ provide an equivalent description to the successfully used descriptors: $F_{\xi}, J_{\xi}$, Eqs. 1 and 2. The goal of this present work is to apply existing conceptual DFT apparatus to show an alternative formulation of $F_{\xi}, J_{\xi}$ 
and their characteristic points on the IRC. An effort is made to escape the tyranny of the finite difference approximation, inevitable in any calculations involving ionization energy $(I)$ and electron affinicty $(A)$, thus also the chemical potential $\mu=1 / 2(I+A)$ and global hardness, $\eta=(I-A)$. Specifically, the relation between the global softness $\left(S=\eta^{-1}\right)$ and the electronic dipole polarizability changes on the IRC have been studied computationally for the model reaction [8] $\mathrm{HF}+\mathrm{CO} \rightarrow \mathrm{HCOF}$.

\section{The method}

Theoretical background

For the purpose of the reaction path analysis, the unique IRC coordinate is specified as $\xi$. Assuming the supermolecule approach to whatever reactant configuration may be, the DFT operational regime is $N=$ const, i.e., the closed system or canonical ensemble approach. The electronic energy $E(\xi)$, chemical potential, global hardness and global softness will vary with the reaction progress as a result of variable nuclear positions. To simplify the formalism, the concise notation for the derivatives is used throughout: $\mu=E^{(N)}, \eta=E^{(N N)}$, etc. The reaction force becomes $[16,17]$ :

$F_{\xi}=-\sum_{i} \frac{\partial E}{\partial \mathbf{R}_{i}} \cdot \frac{d \mathbf{R}_{i}}{d \xi}=\sum_{i} \mathbf{F}_{i} \cdot \frac{d \mathbf{R}_{i}}{d \xi}$.

Here $\mathbf{F}_{\mathrm{i}}$ stands for the Hellman-Feynman force acting on $i$ th nucleus, the summation may be limited to atoms relevant for the reaction $\left(\frac{d \mathbf{R}_{i}}{d \xi} \neq 0\right)$. By the same token, the derivative of the chemical potential provides an alternative formulation for the electronic flux (Eq. 2):

$J_{\xi}=\frac{d \mu}{d \xi}=-\frac{d F_{\xi}}{d N}=-\sum_{i} \frac{d \mathbf{F}_{i}}{d N} \cdot \frac{d \mathbf{R}_{i}}{d \xi}=\sum_{i} \boldsymbol{\Phi}_{i} \cdot \frac{d \mathbf{R}_{i}}{d \xi}$.

The derivative $d / d N$ is taken at constant external potential, hence at $\mathbf{R}_{i}=$ const; the symbol $\boldsymbol{\Phi}_{i}=-E^{(R N)}$ has been used for the nuclear reactivity index [12]. At the finite difference approximation this index is calculated from the Hellman-Feynman forces in respective ions:

$\boldsymbol{\Phi}_{i}=-\frac{1}{2}\left(\mathbf{F}_{i}^{+}-\mathbf{F}_{i}^{-}\right)$.

Hence:

$J_{\xi}=-\frac{1}{2}\left(F_{\xi}^{+}-F_{\xi}^{-}\right)$

$F_{\xi}^{+}, F_{\xi}^{-}$are reaction forces calculated for the ionized forms of the molecule. Equations 4 and 6 provide the electronic flux value identical to the original definition (Eq. 1) within the finite difference approximation only if the summation extends to all atoms in the system (see Discussion and conclusions).

The derivative of hardness and softness is calculated by exploring the nuclear stiffness index [12] $\mathbf{G}_{i}=E^{(R N N)}$ :

$\mathbf{G}_{i}=\frac{1}{2}\left(\mathbf{F}_{i}^{+}+\mathbf{F}_{i}^{-}\right)-\mathbf{F}_{i}$

$\frac{d \eta}{d \xi}=-\frac{d^{2} F_{\xi}}{d N^{2}}=-\sum_{i} \frac{d^{2} \mathbf{F}_{i}}{d N^{2}} \cdot \frac{d \mathbf{R}_{i}}{d \xi}=-\sum_{i} \mathbf{G}_{i} \cdot \frac{d \mathbf{R}_{i}}{d \xi}$.

Using identities provided by Ordon [17], the derivative of global softness $S$ becomes:

$\frac{d S}{d \xi}=-S^{2} \sum_{i} \mathbf{G}_{i} \cdot \frac{d \mathbf{R}_{i}}{d \xi}$.

The electronic dipole polarizability is by definition the second energy derivative over the external field $\varepsilon: \alpha_{e}=\frac{d^{2} E}{d \varepsilon^{2}}$. Vela and Gazques [18] first demonstrated a linear relationship between $\alpha_{e}$ and the global softness of a system. Its variation on the IRC has not yet been the subject of an analysis.

Critical points on the IRC

The reaction force extreme is found through the derivative:

$$
\begin{aligned}
\frac{d F_{\xi}}{d \xi} & =\sum_{i} \frac{\partial F_{\xi}}{\partial \mathbf{R}_{i}} \cdot \frac{d \mathbf{R}_{i}}{d \xi}=\sum_{i} \frac{\partial}{\partial \mathbf{R}_{i}}\left(\sum_{j} \mathbf{F}_{j} \cdot \frac{d \mathbf{R}_{j}}{d \xi}\right) \cdot \frac{d \mathbf{R}_{i}}{d \xi} \\
& =\sum_{i, j} \frac{\partial \mathbf{F}_{j}}{\partial \mathbf{R}_{i}} \cdot \frac{d \mathbf{R}_{j}}{d \xi} \cdot \frac{d \mathbf{R}_{i}}{d \xi}
\end{aligned}
$$

$\frac{\partial}{\partial \mathbf{R}_{i}} \frac{d \mathbf{R}_{j}}{d \xi}=\frac{\partial \delta_{i j}}{\partial \xi}=0$ since $\mathbf{R}_{j}$ are independent variables. The derivative $\frac{\partial \mathbf{F}_{j}}{\partial \mathbf{R}_{i}}=k_{i j}=E^{(R R)}$ is an element of the force constants matrix [16] and Eq. 10 becomes identical to the reaction force constant, as discussed by other authors [19, 20]:

$\frac{d F_{\xi}}{d \xi}=\sum_{i, j} k_{i j} \frac{d \mathbf{R}_{j}}{d \xi} \frac{d \mathbf{R}_{i}}{d \xi}=k_{\xi}$.

The conditions for the extreme reaction force is then $k_{\xi}=0$, as expected.

The critical points for the electronic flux (Eq. 1) result formally by taking a derivative of Eq. 4 :

$$
\frac{d J_{\xi}}{d \xi}=\sum_{i, j} \lambda_{i j}(\xi) \cdot \frac{d \mathbf{R}_{j}}{d \xi} \cdot \frac{d \mathbf{R}_{i}}{d \xi}=\frac{1}{2}\left[k_{\xi}^{+}-k_{\xi}^{-}\right]
$$

$k_{\xi}^{+}, k_{\xi}^{-}$are reaction force constants calculated for the ionized species, respectively, $\lambda_{i j}$ stands for the component of the 
mode softening index introduced in earlier works from this laboratory: $[8,21]$

$$
\lambda_{i j}=\frac{\partial \Phi_{j}}{\partial \mathbf{R}_{i}}=\frac{\partial k_{i j}}{\partial N} \cong \frac{1}{2}\left[k_{i j}^{+}-k_{i j}^{-}\right] .
$$

The critical points on the electronic flux profile are determined by the dependence of the reaction force constants on the number of electrons $k_{\xi}(N)$. The extreme hardness and softness appear at the same point on the IRC (Eqs. 8 and 9) [12]:

$\frac{d \eta}{d \xi}=F_{\xi}-\frac{1}{2}\left(F_{\xi}^{+}+F_{\xi}^{-}\right)=0$.

The critical points for the reaction force $F_{\xi}$ (Eq. 10), electronic flux $J_{\xi}$ (Eq. 12) and hardness/softness appear to be determined independently, by different factors.

\section{Electron dipole polarizability}

The extreme of the electronic part of the dipole polarizability $\left(\mathbf{R}_{\mathrm{i}}=\right.$ const $)$ is found by definition from the derivative:

$$
\begin{aligned}
\frac{d \underline{\underline{\boldsymbol{\alpha}}}_{e}}{d \xi} & =\frac{d}{d \xi} \frac{d^{2} E}{d \boldsymbol{\varepsilon}^{2}}=\frac{d^{2} F_{\xi}}{d \boldsymbol{\varepsilon}^{2}}=\frac{d^{2}}{d \boldsymbol{\varepsilon}^{2}} \sum_{i} \mathbf{F}_{i} \cdot \frac{d \mathbf{R}_{i}}{d \xi} \\
& =\sum_{i} \frac{d^{2} \mathbf{F}_{i}}{d \boldsymbol{\varepsilon}^{2}} \cdot \frac{d \mathbf{R}_{i}}{d \xi} .
\end{aligned}
$$

The derivative of force may be expressed using the Hellman-Feynman force at $i$ th nucleus:

$\mathbf{F}_{i}=\mathbf{F}_{i}^{n-n}+\int \rho(\mathbf{r}) \cdot \boldsymbol{\varepsilon}_{i}(\mathbf{r}) d \mathbf{r}$ where $\boldsymbol{\varepsilon}_{\mathrm{i}}(\mathbf{r})=-\frac{\mathrm{Z}_{\mathrm{i}}\left(\mathrm{R}_{\mathrm{i}}-\mathbf{r}\right)}{\left|\mathrm{R}_{\mathrm{i}}-\mathbf{r}\right|^{3}}$.

Hence:

$\frac{d \mathbf{F}_{i}}{d \boldsymbol{\varepsilon}}=\int \boldsymbol{\alpha}(\mathbf{r}) \boldsymbol{\varepsilon}_{i}(\mathbf{r}) d \mathbf{r}$

and

$\frac{d \underline{\underline{\boldsymbol{\alpha}}}_{e}}{d \xi}=\sum_{i} \frac{d \mathbf{R}_{i}}{d \xi} \cdot \int \underline{\underline{\beta_{e}}}(\mathbf{r}) \boldsymbol{\varepsilon}_{i}(\mathbf{r}) d \mathbf{r}$.

The local polarization vector $\boldsymbol{\alpha}(\mathbf{r})$ has been introduced by Komorowski et al. $[22,23]$ as a tool to express the polarization justified Fukui functions; $\underline{\beta}_{e}(r)$ is computable local electronic hyperpolarizability tensor. The formal result (Eq. 18) indicates, that the meaningful contributions to the derivative of the electronic dipole polarizability on the IRC that determines the polarizability extreme are due to atoms

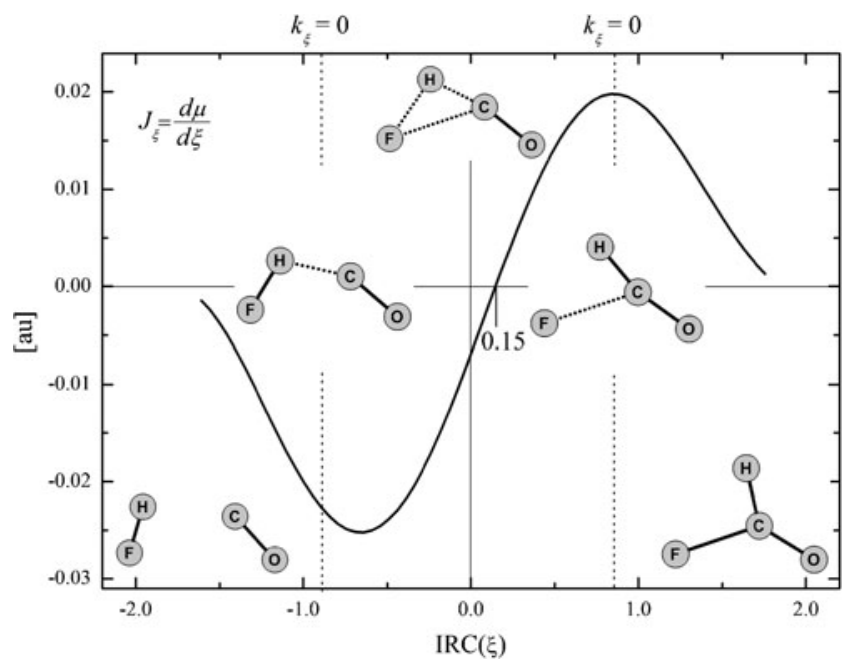

Fig. 1 Reaction electronic flux $J_{\xi}$ calculated from the definition (Eq. 1). The arrangement of atoms is shown at the characteristic reaction points

relevant for the reaction progress only $\left(\frac{d \mathbf{R}_{i}}{d \xi} \neq 0\right)$. The same is evident in $\frac{d \eta}{d \xi}$ and $\frac{d S}{d \xi}$ derivatives, Eqs. 8 and 9.

Calculations and results

The reaction selected for this study has been already been studied [8]:

$\mathrm{HF}+\mathrm{CO} \rightarrow \mathrm{HCOF}$.

Its IRC energy profile has been reproduced by the standard IRC procedure at the MP2 level using the 6-311++ $\mathrm{G}(3 d f, 3 p d)$ basis set and the Gaussian 09 code [24]. Ionization energy $(I)$ and electron affinity $(A)$ were calculated for the geometry determined in each point on the reaction path.

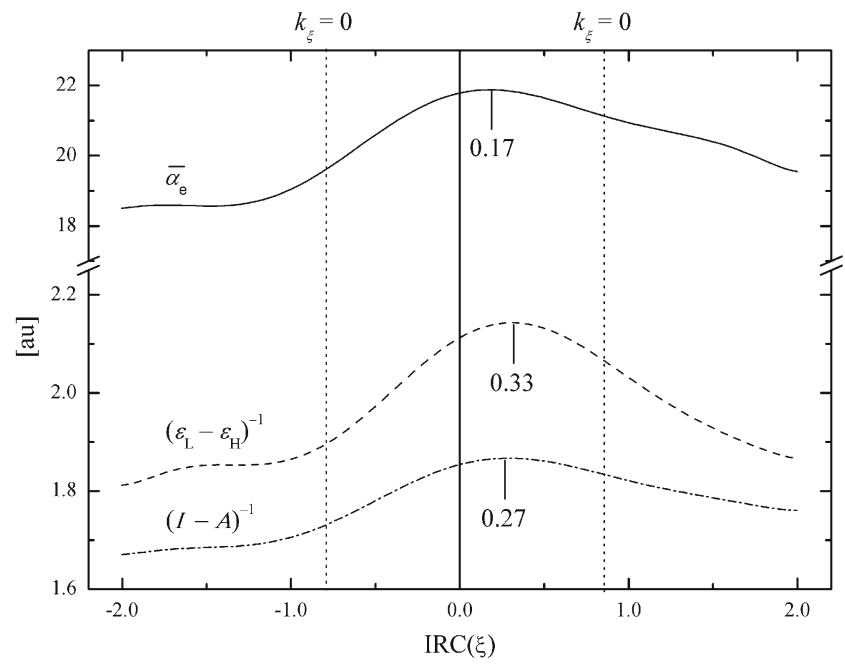

Fig. 2 Variation of the electron dipole polarizability $\left(\bar{\alpha}_{e}\right)$ and global softness calculated in two approximations: $(I-A)^{-1}$ and $\left(\varepsilon_{\mathrm{L}}-\varepsilon_{\mathrm{H}}\right)^{-1}$. Position of the maximum has been marked on each curve 
The $\varepsilon_{H O M O}$ and $\varepsilon_{L U M O}$ energies were found from the HF optimization. Electron dipole polarizability has been calculated using the finite field approximation, the trace of the polarization tensor has been calculated for each point.

The reaction electronic flux calculated for the reaction is presented in Fig. 1. The variable arrangement of atoms on the IRC is shown in five characteristic points. Within the reaction region (limited by the points where the reaction force constant is zero), only minor geometry change occurs, the hydrogen moving slightly off the fluorine atom (see also the TOC picture).

Electron dipole polarizability on the reaction path is shown in Fig. 2. The results of quantum chemical computation for points on the IRC have been approximated by a polynomial curve (8th degree) to produce smooth and differentiable functions. The extreme points (maxima) have been determined with the accuracy of this analytic approximation to the computed points.

\section{Discussion and conclusions}

Two results of the present work represent a novel contribution to the reaction path analysis. The calculated electron dipole polarizability corroborates an earlier finding of the softest state in this reaction [8], away from its transition state. However, the present result is obtained beyond the limiting finite difference approximation, which was also tested here in two typical modifications, exploring the computed $I(\xi)$ and $A(\xi)$ results (MP2 level) or the orbital energies $\varepsilon(\xi)$ on the HF level. The qualitative agreement between the results of these two approaches can be explained on the ground of the relation between the softness $(S)$ and the electron polarizability $\left(\alpha_{e}\right)$, first indicated in the work from this laboratory [25] and elaborated by Vela and Gazques [18] to produce the rigorous result:

$\underline{\underline{\boldsymbol{\alpha}_{e}}}=-S\left[\left(\int f(\mathbf{r}) \mathbf{r} d \mathbf{r}\right)^{2}-\int f(\mathbf{r}) \mathbf{r r} d \mathbf{r}\right]$.

This result may be reformulated by introducing the global properties of the system instead of the Fukui function $f(\mathbf{r})=$ $d \rho(\mathbf{r}) / d N$ : electronic parts of the dipole moment $\left(\mathbf{M}_{e}\right)$ and of the quadruple moment of the system $\left(\mathbf{Q}_{e}\right)$. The scalar electron dipole polarizability is then:

$\alpha_{e}=-\frac{S}{3} \sum_{i=x, y, z}\left[\left(\frac{d M_{e, i}}{d N}\right)^{2}-\frac{d Q_{e, i i}}{d N}\right]$.

It is interesting to note in Eq. 20, that the polarizability calculated on the IRC with no relation to differentiation over the number of electrons $N$ is still roughly parallel to $S=(I-$ $A)^{-1}$, as if the variations of the electron dipole and quadruple moments were immaterial. Theoretical result for the derivative of $\alpha_{e}$ (Eq. 18) indicates, however, that this relation is not granted, especially when softness is calculated from the orbital energies, $\left(\varepsilon_{\mathrm{L}^{-}} \varepsilon_{\mathrm{H}}\right)^{-1}$. The non-zero contribution to $d \alpha_{e} d \xi$ (Eq. 16-18) comes from atoms relevant to the reaction in question: either close to the reaction center (large field $\varepsilon_{i}$ ) or significant for the reaction coordinate (non vanishing $d R_{i} / d \xi$ ). On the contrary, the global softness $S$ may be dominated by the energies of orbitals possibly located far away from the reaction center.

Numerical result for the electronic flux (Fig. 1) compared to the softness/polarizability diagram (Fig. 2) reveals the coincidence: electronic flux is zero at the softest state. This is not unexpected, though never observed so far. The electronic flux is zero at the chemical potential minimum; apparently this is a property of a softest state, not necessarily the transition state. A quick search of a softest state is possible by means of the result in Eq. 6 for zero flux, $F_{\xi}^{+}=F_{\xi}^{-}$or, else, from Eq. 14 for the softest state $F_{\xi}=\frac{1}{2}\left(F_{\xi}^{+}+F_{\xi}^{-}\right)$. The interesting property of a softest state/zero flux is then the reaction force independent of the moderate ionization of a reacting system: $F_{\xi}^{+}=F_{\xi}^{-}=F_{\xi}$. Electronic flux extremes in Fig. 1 do not coincide with $k_{\xi}=0$; this is natural from the result of Eq. 12.

The theoretical result for the reaction electronic flux demonstrates a similar feature, here even more important. The $J_{\xi}$ itself (Eq. 6) and its derivative (Eq. 12) contains contributions from atoms significant for the reaction coordinate only $\left(d \mathbf{R}_{i} / d \xi \neq 0\right)$; this result appears to be independent of the applied approximation level. For the reactions in large molecular systems calculation of the electronic flux and its derivatives from global properties exclusively (Eq. 2) may produce results irrelevant for the reaction in question, when either $I$, or $A$, or both are dominated by contributions from some far away regions in a system. The result given by Eq. 4, even if used in the finite difference approximation (Eq. 6), describes precisely the properties of the reaction center, including specific contributions from the rest of the molecule, if they are relevant for the reacting $\operatorname{mode}\left(d \mathbf{R}_{i} / d \xi \neq\right.$ $0)$. This appears to be a superior point of view as compared to the classical concept (Eq. 1).

Acknowledgments The work was financed by a statutory activity subsidy from the Polish Ministry of Science and Higher Education for the Faculty of Chemistry of Wrocław University of Technology. The use of resources of Wrocław Center for Networking and Supercomputing (WCSS) is gratefully acknowledged.

Open Access This article is distributed under the terms of the Creative Commons Attribution License which permits any use, distribution, and reproduction in any medium, provided the original author(s) and the source are credited. 


\section{References}

1. Toro-Labbé A (1999) J Phys Chem A 103:4398

2. Herrera B, Toro-Labbé A (2007) J Phys Chem A 111:5921

3. Echegaray E, Toro-Labbé A (2008) J Phys Chem A 112:11801

4. Flores-Morales P, Gutiérrez-Oliva S, Silva E, Toro-Labbé A (2010) J Mol Struct Theochem 943:121

5. Cerón ML, Echegaray E, Gutiérrez-Oliva S, Herrera B, ToroLabbé A (2011) Sci China Chem 54:1982

6. Torrent-Sucarrat M, Luis JM, Duran M, Solà M (2004) J Chem Phys 120:10914

7. Torrent-Sucarrat M, Geerlings P, Luis JM (2007) Chem Phys Chem 8:1065

8. Ordon P, Tachibana A (2007) J Chem Phys $126: 234115$

9. Ordon P, Tachibana A (2005) J Mol Model 11:312

10. Parr RG, Yang W (1989) Density-functional theory of atoms and molecules. Oxford University Press, New York

11. Geerlings P, De Proft F, Langenaeker W (2003) Chem Rev 103:1793

12. Ordon P, Komorowski L (1998) Chem Phys Lett 292:22

13. Ordon P, Komorowski L (2005) Int J Quant Chem 101:703

14. Komorowski L, Ordon P (2004) Int J Quant Chem 99:153

15. Miller WH, Handy NC, Adams JE (1980) J Chem Phys 72:99

16. Wilson EB Jr, Decius JC, Cross PC (1980) Molecular vibrations. Dover, New York

17. Ordon P (2003) Dissertation, Wrocław University of Technology, Wrocław
18. Vela A, Gazquez JL (1990) J Am Chem Soc 112:1490

19. Jaque P, Toro-Labbé A, Politzer P, Geerlings P (2008) Chem Phys Lett 456:135

20. Yepes D, Murray JS, Politzer P, Jaque P (2012) Phys Chem Chem Phys 14:11125

21. Komorowski L, Ordon P (2001) Thoer Chem Acc 105:338

22. Komorowski L, Lipiński J, Szarek P (2009) J Chem Phys 131:124120

23. Komorowski L, Lipiński J, Szarek P, Ordon P (2011) J Chem Phys 135:014109

24. Frisch MJ, Trucks GW, Schlegel HB, Scuseria GE, Robb MA, Cheeseman JR, Scalmani G, Barone V, Mennucci B, Petersson GA, Nakatsuji H, Caricato M, Li X, Hratchian HP, Izmaylov AF, Bloino J, Zheng G, Sonnenberg JL, Hada M, Ehara M, Toyota K, Fukuda R, Hasegawa J, Ishida M, Nakajima T, Honda Y, Kitao O, Nakai H, Vreven T, Montgomery JA Jr, Peralta JE, Ogliaro F, Bearpark M, Heyd JJ, Brothers E, Kudin KN, Staroverov VN, Kobayashi R, Normand J, Raghavachari K, Rendell A, Burant JC, Iyengar SS, Tomasi J, Cossi M, Rega N, Millam JM, Klene M, Knox JE, Cross JB, Bakken V, Adamo C, Jaramillo J, Gomperts R, Stratmann RE, Yazyev O, Austin AJ, Cammi R, Pomelli C, Ochterski JW, Martin RL, Morokuma K, Zakrzewski VG, Voth GA, Salvador P, Dannenberg JJ, Dapprich S, Daniels AD, Farkas Ö, Foresman JB, Ortiz JV, Cioslowski J, Fox DJ (2009) Gaussian 09, Revision A.02. Gaussian Inc, Wallingford

25. Komorowski L (1987) Chem Phys 114:55 